\title{
Medication Error
}

National Cancer Institute

\section{Source}

National Cancer Institute. Medication Error. NCI Thesaurus. Code C156627.

An error associated with the prescribing, dispensing, or giving/taking a medication by a

healthcare professional, patient, or consumer that causes or leads to inappropriate

medication use or patient harm. 